\title{
Editorial
}

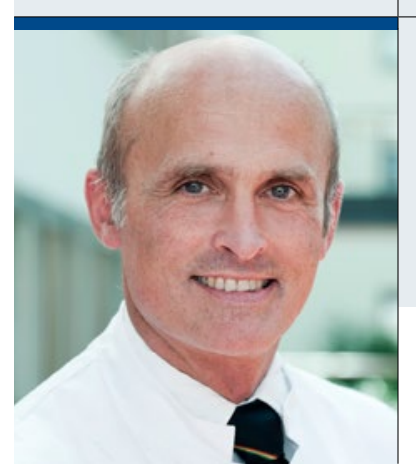

"In der Niere gibt es Veränderungen und Krankheitsbilder,

die Urologen im Laufe eines erfüllten beruflichen Daseins

immer fremder werden."

\section{Was ist eigentlich jenseits der Henleschen Schleife los?}

st es nicht erstaunlich, wie sehr man sich in einem einzigen Organ so fremd werden kann wie in der Niere? Urologen - die Chirurgen an dieser Struktur - betrachten sie ganz anders als Nephrologen: Während Urologen sie von außen, also eher makroskopisch, oder seit 20 Jahren auch gerne von innen, mithin endoskopisch, aber immer noch von der Oberfläche her ansehen und beurteilen, sind die $\mathrm{Ne}$ phrologen eher in der Niere drinnen in ihrem Milieu. Dort gibt es Veränderungen und Krankheitsbilder, die Urologen im Laufe eines erfüllten beruflichen Daseins immer fremder werden. Dabei haben sich die ersten Auffälligkeiten in der Diagnostik seit unserem Studium nicht verändert, und so schien es uns angebracht, diese Erinnerungen zu reaktivieren. Tobias Meyer weist zwar auf die modernste Forschung im Bereich der Urindiagnostik hin, bricht aber noch einmal die Lanze für den Urinteststreifen. Besonders interessant ist, dass aus dem Ergebnis des Teststreifens heute ganz andere Konsequenzen gezogen werden als früher, weswegen die Lektüre seines Beitrags zur Proteinurie empfohlen wird.

Mit der Mikrohämaturie ist es so ein Kreuz: Einerseits läuft zwar die Hälfte unserer Mitbürger mit dieser Diagnose herum, ohne dass sich irgendetwas dahinter verbirgt, andererseits gibt es Malignome im urologischen Bereich, die bereits früh mit einer Mikrohämaturie auf sich aufmerksam machen. Jedoch liegen diese Malignome nur im Promillebereich. In Anbetracht limitierter Ressourcen ist es also eine verantwortungsvolle Aufgabe, die eine Gruppe aus der anderen herauszufischen.

Oliver Gross - nicht verwandt und nicht verschwägert - wendet sich den uronephrologischen Krankheitsbildern zu. Zwar weist er bereits im ersten Absatz seines Artikels darauf hin, dass nicht jede Proteinurie oder Mikrohämaturie beim Nephrologen vorgestellt werden muss. Jedoch gibt er genau die Hinweise, die wir Urologen brauchen, um zu wissen, wann der Kontakt zum Nephrologen hergestellt werden soll und unterstreicht die wichtige Aufgabe der Urologen als Lotsen in diesem Bereich. Die genannten Anhaltspunkte sind in gleichem Maße schlicht wie hilfreich und ganz besonders nephroprotektiv.

Eher vertraut ist der Urologe mit einem anderen Krankheitsbild in der Niere, der Urolithiasis. Steigende Zahlen in diesem Bereich durch schlechter werdende Ernährungsgewohnheiten und viele $\mathrm{Zu}$ wanderer aus an Steinen reichen Regionen rücken die Bedeutung der Steinanalyse wieder in ein besonderes Licht. Roswitha Siener aus Bonn berichtet hierzu aus „ihrem“ Spezialbereich, in dem sie wie kaum eine andere zu Hause ist.

Wenn der Kliniker zugelangt und den Patienten möglicherweise von seinem Tumor befreit hat, ist das Leiden nicht beendet. Michael Zellner hat als Rehabilitationsmediziner tiefen Einblick in diese Problematik. Er lässt uns an seinem umfangreichen Wissen teilhaben und beschäftigt sich im dritten Teil seiner Serie mit der Bedeutung der Rehabilitation und Nachsorge nach Zystektomie. Nach diesem Artikel weiß man, wieso sich der Rehabilitationsmediziner auch als Präventionsmediziner versteht.

Ein Schmankerl verdanken wir schließlich Daniela Drescher. Sie beschäftigt sich in ihrem Artikel mit Akupunktur in der Urologie. Patienten fragen danach, und so sind ihre Informationen $\mathrm{zu}$ dem Thema sehr hilfreich, wenn man mitreden will.

Unsere Beirätin Roswitha Siener hatte die Verantwortung für die Zusammenstellung des Schwerpunkts dieser URO-NEWS „Erkrankungen der Niere" übernommen, und wir bedanken uns für dieses unseres Erachtens gelungene Vorhaben!

Viel Spaß bei der Lektüre

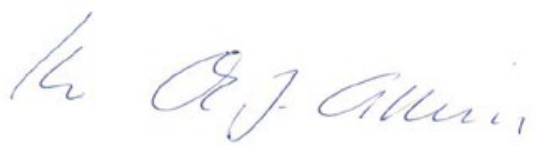

